\title{
LA POLICROMÍA DE LAS YESERÍAS DEL ORATORIO DE LA MADRAZA DE YŪSUF I, GRANADA. PRIMERAS APORTACIONES DEL ESTUDIO DE MATERIALES PARA LA LOCALIZACIÓN DE ZONAS ORIGINALES Y AÑADIDOS*
}

\author{
THE POLYCHROMY OF THE PLASTERWORK OF THE \\ ORATORY OF THE YŪSUF I MADRASA IN GRANADA. \\ INITIAL CONTRIBUTIONS TOWARDS THE IDENTIFICATION \\ OF THE ORIGINAL AREA AND LATER ADDITIONS
}

\author{
Ana García Bueno \\ Víctor J. Medina Flórez \\ Alicia González Segura \\ Universidad de Granada
}

\section{Introducción}

El Palacio de la Madraza constituye un edificio de singular interés en el contexto de la arquitectura granadina e hispana [...] Su nombre deriva de la llamada, ya en época Medieval, Madrasa Yūsufiyya o Madrasa Nașriyya, fundación realizada el año 1349, auspiciada por su ministro (hââyib) Riḍ̂ān, bajo el gobierno del sultán nazarí Yusuf I. Fue esta madraza la segunda creada en alAndalus y la única como fundación docente estatal de estas características ${ }^{1}$.

* Este trabajo se ha desarrollando gracias a la financiación de dos Proyectos de Investigación, "El color de los revestimientos murales en la arquitectura andalusí", Ministerio de Educación, Política Social y Deporte (HUM 2005-03995/ARTE), y "Estudio y conservación de la decoración arquitectónica de tradición islámica", Proyecto de Excelencia de la Junta de Andalucía (HUM-02829), y al contrato con el Vicerrectorado de Patrimonio, Infraestructura y Equipamiento de la Universidad de Granada. Además queremos hacer constar nuestro agradecimiento a la entonces Vicerrectora, Elena Díez Jorge, por encomendarnos su realización y al Dr. Parra Crego, de la Universidad Alfonso X el Sabio, por el estudio de aglutinantes.

${ }^{1}$ Cruz Cabrera, J.P. y Gómez-Moreno Calera, J.M., "Estudio histórico-artístico del palacio de la Madraza, antigua casa del Cabildo de Granada", en R. López Guzmán y Mª E. Díez Jorge (eds), La Madraza: pasado presente y futuro, Granada, 2007, 43. 


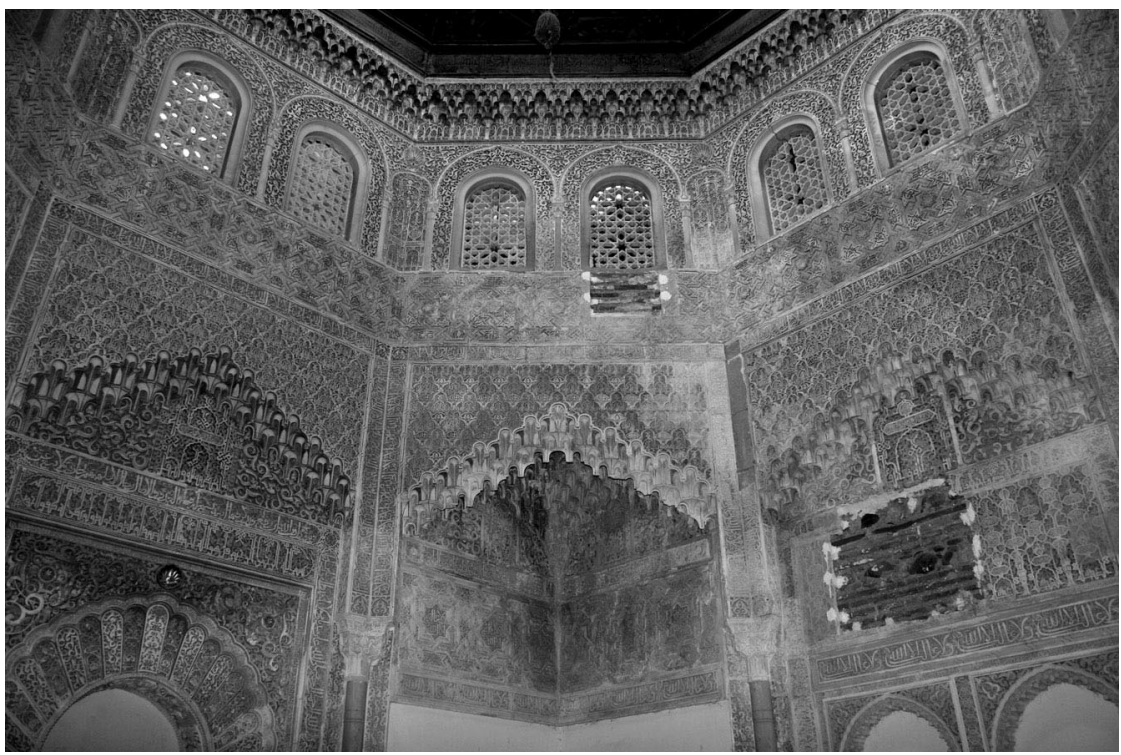

Figura 0. Imagen general de la Madraza de Yūsuf I, Granada

Al afrontar este trabajo nuestro principal objetivo fue recoger toda la información material disponible para, posteriormente, poder orientar la restauración con los criterios más adecuados y poder seleccionar tratamientos absolutamente compatibles con los materiales originales.

Dada la brevedad de este artículo, nos centraremos en el estudio de la policromía, cuestión que nos parece sumamente interesante ya que nos permitirá hacer aproximaciones sobre zonas originales y añadidos posteriores. Dicho objetivo se plantea después de comprobar, tanto por el informe histórico como por la observación detallada de la obra, que el oratorio ha tenido intervenciones importantes en las que, no sólo se hicieron reparaciones, sino que también se adaptó el espacio a las necesidades y gustos de cada momento. En este sentido, debemos resaltar la adaptación realizada a finales del siglo XIX en la que se aplicó una capa de color que cubría prácticamente toda la superficie decorada. Sin embargo, después de hacer una revisión completa se comprobó que, aunque efectivamente la mayor parte de la superficie de yeserías estaba repintada, había zonas que parecían originales. Así mismo, se observaron zonas en las que debajo de las 
capas de color de la restauración se conservaban restos de color original. Los criterios de intervención, hasta bien entrado el siglo xx, no requerían la diferenciación entre las partes originales y los añadidos, lo que dificulta la interpretación de resultados.

En éste, como en otros muchos casos, el conocimiento de los materiales y su disposición puede ser definitivo para detectar diferencias objetivas entre los materiales correspondientes a las distintas intervenciones.

\section{Metodología}

Una vez que se había revisado toda la superficie de yeserías, se procedió a la toma de muestras ${ }^{2}$. Ésta se llevó a cabo en el momento en que el examen organoléptico estaba muy avanzado y, por tanto, nos permitía, con un número de extracciones relativamente reducido, conseguir un máximo de información. Aún así, dada la enorme superficie decorada y la diversidad de intervenciones, el número de muestras ha sido muy importante. Se han recogido 120 para el estudio de pigmentos, 27 para el de aglutinantes y 50 para el análisis de morteros.

Con el estudio de materiales se persigue identificar los componentes de los revestimientos, tanto de los morteros (aglomerante, árido y posibles adiciones de materia orgánica) como de la capa de color que los cubre (pigmentos y aglutinantes) ${ }^{3}$.

\section{Estudio de materiales}

En esta publicación nos limitaremos a exponer los resultados de los muros sur y del mihrāb, aunque el proceso de trabajo ha abarcado toda la superficie decorada con yeserías.

2 Para más detalles sobre la metodología, consultar García Bueno, A., Medina Flórez, V.J. y Hernández Pablos, A., "Estudio previo a la restauración de las yeserías del oratorio de la Madraza (metodología y avance de resultados)", en López Guzmán y Díez Jorge, La Madraza, 277-304.

${ }^{3}$ Los métodos seleccionados para el posterior estudio de las muestras tomadas son: para la identificación de material inorgánico: microscopía óptica, microscopía electrónica de barrido y difracción de rayos X; para la identificación de materia orgánica: ensayos de coloración, infrarrojos por transformada de Fourier y métodos cromatográficos, cromatografía de gases o cromatografía líquida de alta presión. 


\subsection{Morteros}

El estudio de morteros muestra claras diferencias en el examen organoléptico previo y en el estudio mediante microscopía óptica, sin embargo, estas diferencias no se vieron reflejadas de forma tan clara en los resultados de los estudios mediante microscopía electrónica de barrido (SEM/EDX) y difracción de rayos X (DRX). Por tanto a la hora de identificar en los morteros zonas originales y añadidos de restauraciones posteriores, los resultados más interesantes nos los proporcionan la observación directa $\mathrm{y}$, sobre todo, el estudio mediante microscopía óptica, ya que estas identificaciones están basadas fundamentalmente en las diferencias de granulometría y estratificación.

Se observan tres tipos característicos: el primer tipo presenta una primera capa de "yeso oscuro", con un alto contenido en tierras, granulometría gruesa, color tostado y textura poco homogénea, sobre el cual se dio una capa de enjalbegado que prepara la superficie para la aplicación del color (Fig. 1). En el segundo se observa un mortero muy blanco sobre el que se aplicó una capa muy fina y discontinua de tierra, ocre/rojiza y, a continuación, un enjalbegado blanco que igualmente prepara la superficie para aplicar la policromía ${ }^{4}$ (Fig. 2). Finalmente encontramos otro tipo de morteros muy homogéneos en color y granulometría (Fig. 3), presentan gran resistencia y cohesión y en ellos no se advierte ninguna estratificación.

Como resultado de este estudio, respecto a los yesos originales, debemos destacar la constatación de la utilización de las dos técnicas originales (yeserías talladas y a molde) en un mismo momento constructivo. Tradicionalmente se ha dicho que en el primer periodo del arte nazarí los yesos se hacían tallados y que, a medida que la superficie decorada aumenta, se tiende a la utilización de moldes. Sin contradecir esta teoría, observamos la convivencia de ambas técnicas, que se explica claramente si se observa que las zonas realizadas mediante talla, son aquellas en las que se comprueba que la decoración no se repite de forma modular y sistemática, sino las que requieren de un desarrollo formal específico para cada localiza-

\footnotetext{
${ }^{4}$ Esta misma estratificación la encontramos también en los fragmentos de yeso estudiados de los depósitos del museo de la Alhambra. García Bueno, A. y Medina Flórez, V.J., "La policromía de los fragmentos de yeso almacenados en los depósitos del museo de la Alhambra", en $16^{\text {th }}$ Internacional Meeting on Heritage Conservation, Valencia, 2006, 1601-1613.
}

Al-Qanțara (AQ) XXXI 1, enero-junio 2010, pp. 245-256 ISSN 0211-3589 


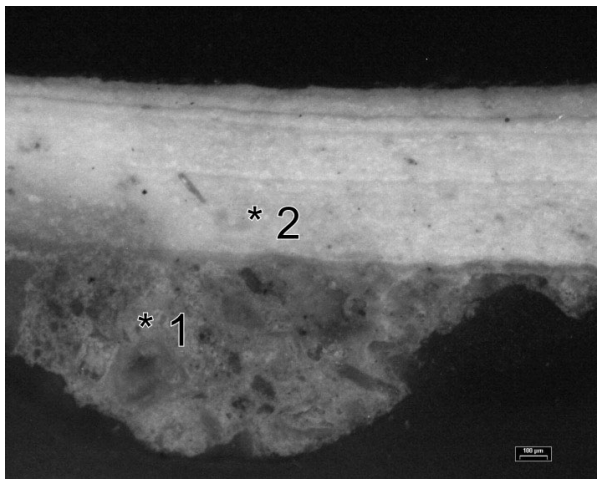

Figura 1. Muestra de mortero correspondiente a una yesería tallada. Microscopía Óptica.

Figura 2. Muestra de mortero correspondiente a una yesería realizada a molde. Microscopía óptica.
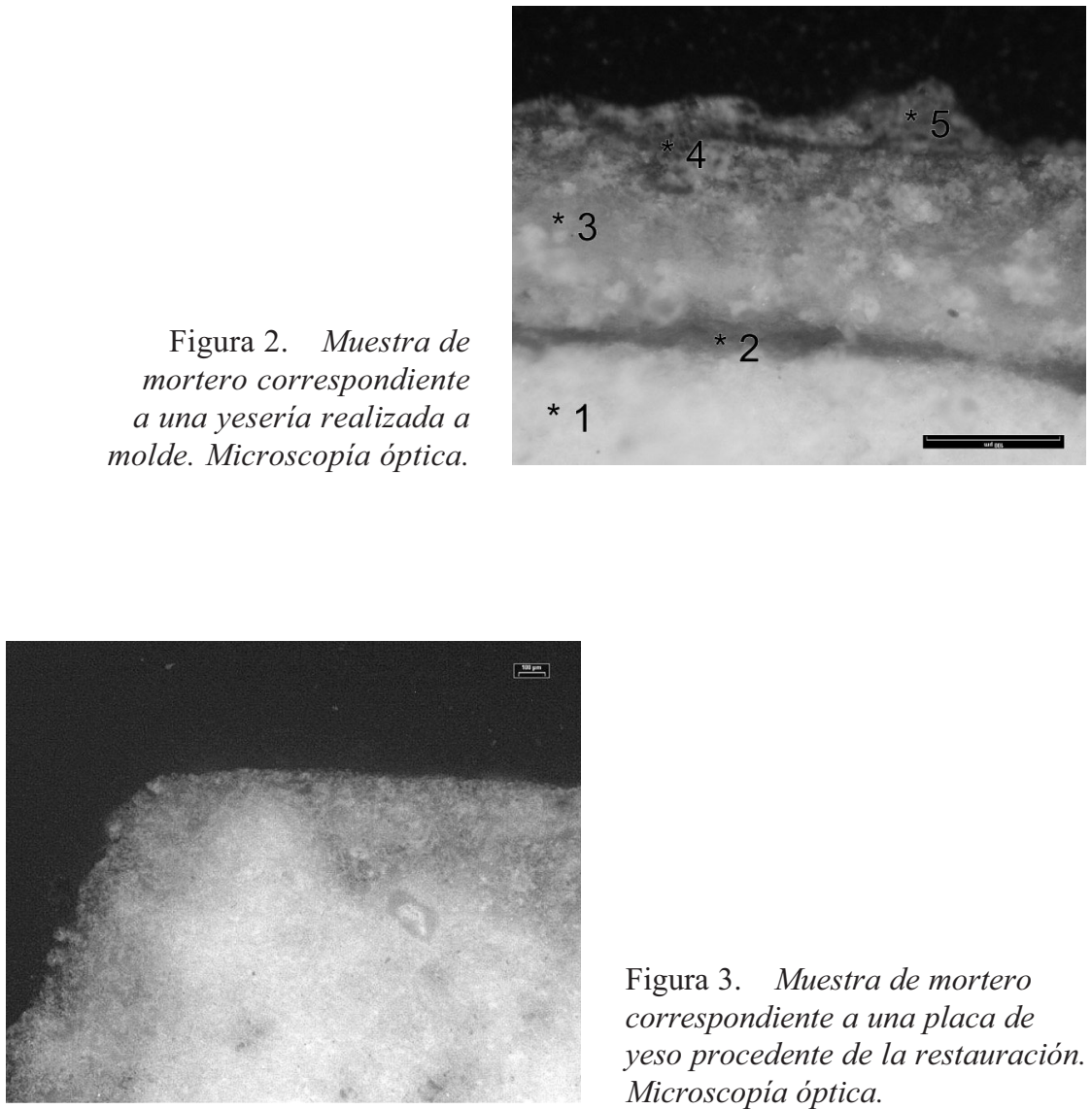

Figura 3. Muestra de mortero correspondiente a una placa de yeso procedente de la restauración. Microscopía óptica.

Al-Qanțara (AQ) XXXI 1, enero-junio 2010, pp. 245-256 ISSN 0211-3589 


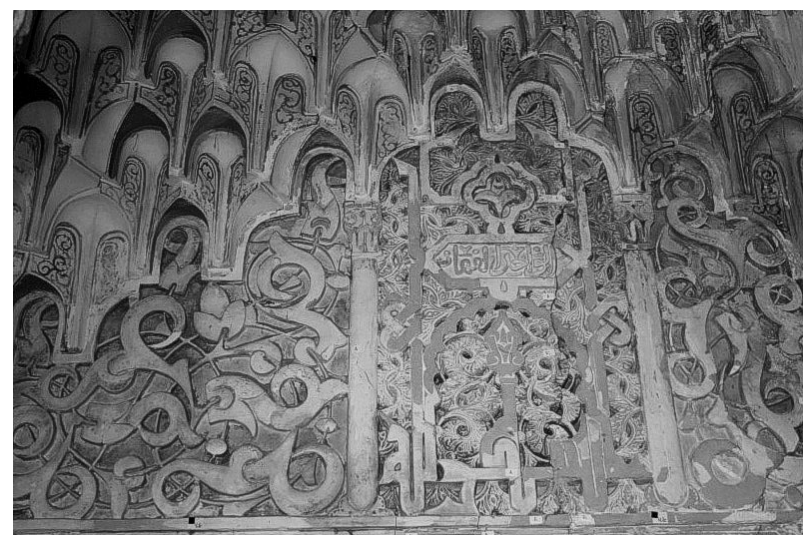

Figura 4. Fragmento de yeserías realizado mediante procedimiento de talla.

ción, por ejemplo en el triángulo decorativo que se forma bajo el arco de mocárabes (Fig. 4). En todos estos casos, los morteros de base coinciden con los tradicionalmente llamados "yesos negros", del primer tipo, muy consistentes y con árido de tierras que les confiere ese color ocre característico (Fig. 1), sobre los cuales se aplican los enjalbegados más blancos $\mathrm{y}$, en su caso, la policromía.

Respecto a los otros dos tipos de morteros, podemos decir que están compuestos por materiales muy similares: yeso, cuarzo y algo de arcillas, estos dos últimos en proporción muy baja. Sin embargo, existen diferencias entre ellos que nos permiten distinguir los yesos de moldes originales de los que se utilizan en las reposiciones posteriores. En los originales, la mayor parte de las muestras presenta una capa discontinua de color tierra ocre/rojiza que podría corresponder a una arcilla o barbotina empleada para el desmoldeo de las piezas (Fig. 2). Sobre esta capa se aplicaría el enjalbegado de yeso que preparaba la superficie para la aplicación de la policromía y que, además, permitía disimular las uniones de las placas de moldes.

A diferencia de éstos, en las yeserías de restauración los morteros son muy homogéneos y no se advierten diferencias significativas en la granulometría (ni en su tamaño, ni en su forma, ni en su color), lo que es propio de los materiales preparados de forma industrial (Fig. 3). Además la ausencia de restos de esa finísima capa de color tierra anaranjada, característica de los yesos originales, nos permite deducir que estos proceden de restauración. 
Figura 5. Fotografia de microscopia óptica. Se pueden apreciar la primera capa de policromía original, a continuación un enjalbegado blanco y, finalmente, la capa de repolicromado moderno. En este caso, el uso de color rojo se mantiene en las dos policromías.

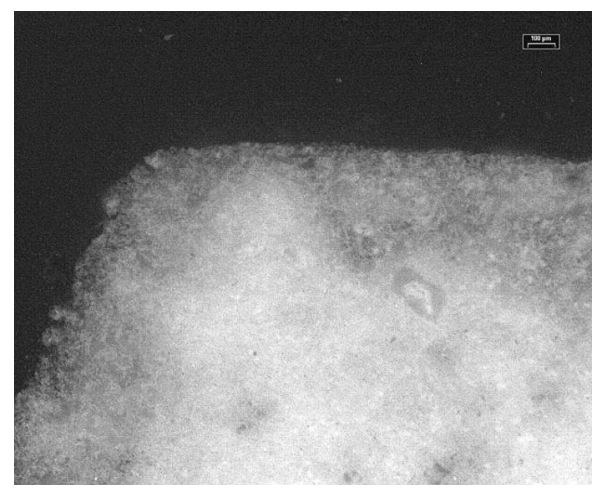

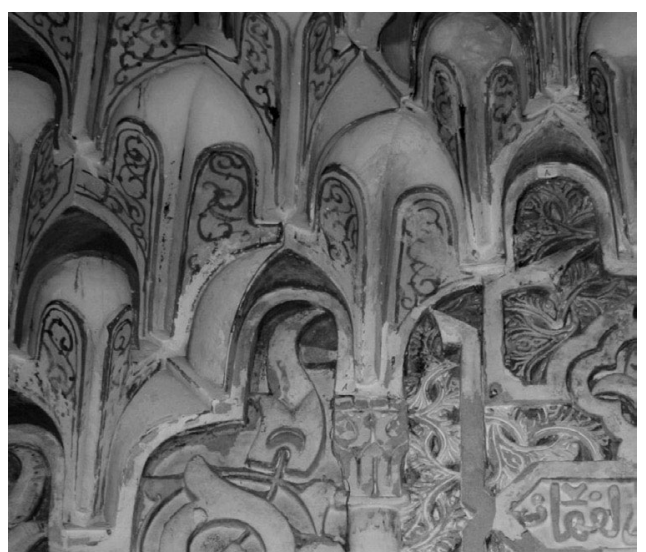

Figura 6. Decoración pintada en los mocárabes, correspondiente a la policromía moderna.

\subsection{Policromía}

En primer lugar debemos recordar que, en la restauración de finales del siglo XIX, se "repolicromó" prácticamente toda la superficie de las yeserías por lo que, a priori, podemos decir que la mayor parte de los pigmentos identificados deben corresponder a dicha restauración. Evidentemente, sobre los morteros de restauración la policromía que aparece será siempre moderna aunque también, sobre los morteros originales, tanto los tallados como los de molde, la policromía puede ser reciente (Fig. 6). Sin embargo, en algunos casos, observamos una superposición de estratos pictóricos que nos indican la existencia de restos de un tratamiento cromático previo (Fig. 7). En unos casos esta "repolicromía" coincide con el color original (Fig. 5) y en otros no. 


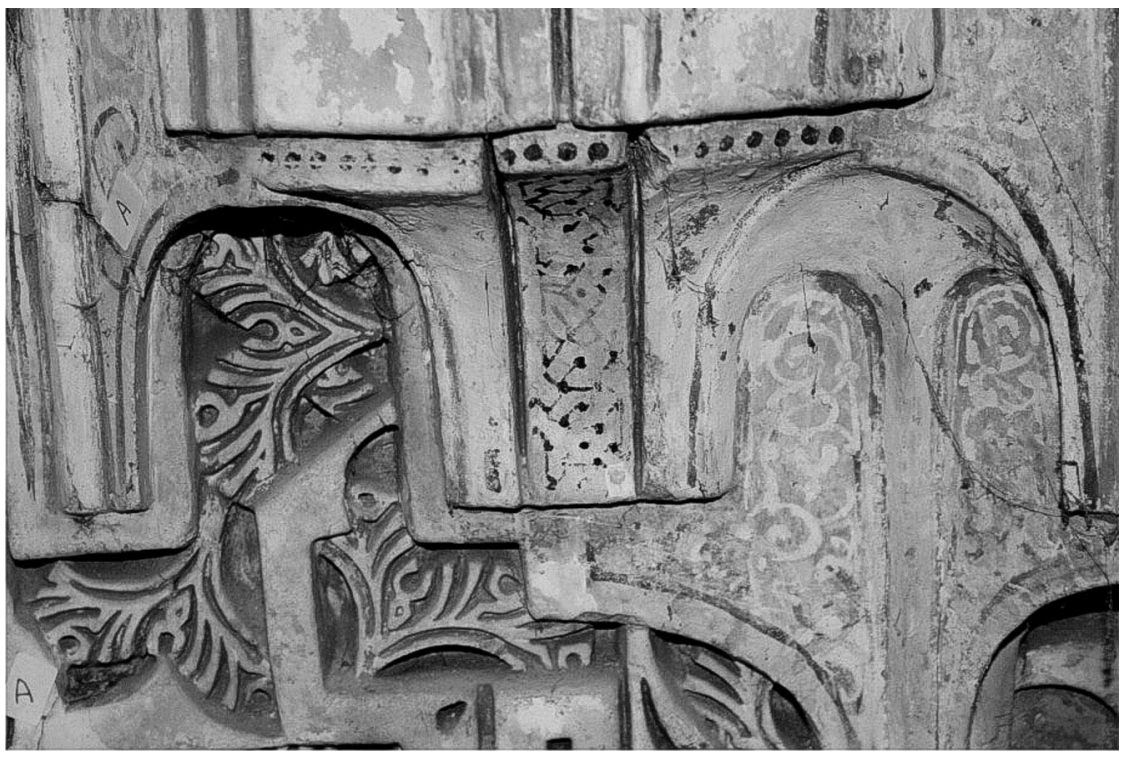

Figura 7. Detalle de los restos de decoración pintada original en los mocárabes

Pigmentos azules: los pigmentos más utilizados para la obtención del color azul son el ultramar (silicato de composición compleja que contiene entre otros elementos $\mathrm{S}, \mathrm{Na}$ ) y la azurita (carbonato básico de cobre), ambos mezclados con barita, con blanco de zinc o de titanio. La barita, aunque es un producto natural conocido desde la antigüedad, se utiliza como pigmento en etapa industrial, empleándolo sólo, denominado como blanco fijo ${ }^{5}$ o litopón, si se mezcla con sulfuro de zinc. Igualmente la presencia en otras muestras de blanco de titanio y de zinc se debe al empleo de pigmentos industriales.

Por otra parte tenemos casos en los que no se detecta ningún elemento atribuible al uso de un pigmento azul mineral; consideramos que el pigmento utilizado es el azul de Prusia, también llamado azul de Berlín (ferrocianuro férrico) ${ }^{6}$. Por tanto ninguno de los pig-

${ }^{5}$ Feller, R.L., "Barium Sulfate, Natural and Synthetic", en idem (ed.), Artists' Pigments. A Handbook of Their History and Characteristics, Nueva York, 1986, 47-64.

${ }^{6}$ A. Orihuela en su publicación sobre las restauraciones de la Alhambra recoge el uso de este pigmento. Orihuela Uzal, A., "La conservación de alicatados en la Alhambra du- 
mentos de las capas pictóricas azules puede ser original, ya que se encuentran mezclados con otros (blancos) de época industrial.

Pigmentos verdes: las capas de color verde están constituidas por una mezcla compleja de pigmentos. Como en el caso anterior, cuando en estas mezclas se identifican pigmentos de etapa industrial, podemos asegurar que, evidentemente, corresponden al "repolicromado" de la restauración. En otras muestras, se detectan pigmentos conocidos desde la antigüedad, generalmente una mezcla de malaquita y verdigris a la que, en ocasiones, se añade un pigmento amarillo para reforzar la tonalidad verdosa (como pigmentos amarillos se emplearon ocre, oropimente o amarillo de plomo y estaño), pero de todas ellas, sólo en un caso podríamos considerarlo original, tanto por la composición del pigmento como por la textura y granulometría del mortero que le sirve de base.

Pigmentos rojos: el pigmento mayoritariamente utilizado es el bermellón (sulfuro de mercurio). Contrariamente a lo que ocurre en otras tonalidades, en el rojo el pigmento no está mezclado. En estos casos el mortero que les sirve de base coincide con las características de los morteros originales, ya sean las de una yesería tallada o las de las yeserías realizadas a molde. En ocasiones se encuentra mezclado con otros pigmentos, tierra roja o tierra roja y minio. Por tanto la mayoría de los pigmentos rojos, por su composición, pueden ser originales. Como en los otros casos, se descarta que sean medievales las muestras en las que se ha identificado litopón y/o barita (Fig. 5).

Pigmentos negros: debemos resaltar la diferencia evidente que se observa en las zonas en las que se ha utilizado este color. Mientras que en algunas de ellas el color aparece muy definido y consistente, en otras el estrato pictórico tiene poquísima densidad, aparece muy agrisado, es poco resistente y por tanto parece corresponder a restos muy degradados de la policromía original. En dos de los casos analizados se identifica negro de hueso (se ha detectado fósforo como elemento característico), sin embargo estas muestras no corresponden a la policromía original ya que se encuentra mezclada con barita. En cambio, en la que ha sido extraída de la zona correspondiente a policromía original se identifica un negro de carbón vegetal.

rante la etapa de Rafael Contreras (1847-1890): ¿modernidad o provisionalidad?”, en J.A. González Alcantud y A. Akmir (eds.), La Alhambra: lugar de la memoria y el diálogo, Granada, 2008, 132-133. 
La única muestra tomada de "capas metálicas", correspondiente al mihrāb, está compuesta por oro, cobre y estaño, aplicada sobre una capa naranja constituida por una fina tierra de óxidos de hierro (un bol que se emplea para preparar la superficie antes de la aplicación del oro). Estas capas corresponden a la policromía moderna aunque los morteros de base, es su mayor parte son originales.

\subsection{Aglutinantes}

En seis de los once registros realizados no se identificó ningún material que pudiese haber servido para fijar el pigmento, lo que sólo se explica por el alto nivel de deterioro que presentan los yesos originales. En las partes mejor conservadas, en las que había restos de policromía original, afortunadamente no se repintó encima, por lo que podemos apreciar su gran calidad y la finura de su trazado. Además, en una muestra de esta misma zona, se pudo identificar goma arábiga como elemento de fijación de la decoración pintada. Su alto nivel de solubilidad en agua y los problemas de filtración de las cubiertas, que han sido muy abundantes, justifican el deteriororo que presenta la policromía y explica que no se identifiquen aglutinantes en muchas de las muestras analizadas.

Por otra parte, encontramos casos en los que se ha identificado únicamente cola animal, en estratos aparentemente originales (inferiores) que corresponden al mihrāb. En otras yeserías estudiadas por nuestro equipo (Cuarto Real de Santo Domingo, Museo de la Alhambra, Casa de la calle San Buenaventura y Fiñana) ${ }^{7}$ los aglutinantes empleados en las capas pictóricas siempre han sido temples magros (solubles en agua), unas veces aglutinados con cola animal y otras con goma arábiga.

En otros puntos en los que la policromía estaba bien conservada, se identifica como aglutinante una mezcla de cola animal, aceite de

${ }^{7}$ García Bueno, A. y Medina Flórez, V.J., "The Nașrid plasterwork at Qubba Dār al-Manjara 1-Kubrà in Granada: Characterisation of materials and techniques", Journal of Cultural Heritage, 5 (2004), 75-89; García Bueno, A., Medina Flórez, V.J. y Hernández Pablos, A., "Intervención de urgencia en una casa musulmana de la calle San Buenaventura $\mathrm{n}^{\circ}$ 2, Albayzín, Granada", en II Congreso del grupo español del IIC. Intervención en Conservación y Restauración, Barcelona, 2005, 359-367; García Bueno y Medina Flórez, "La policromía", 1601-1613. 
linaza y a veces resinas diterpénicas. Este resultado coincide siempre con el uso de pigmentos de etapa industrial, por lo que consideramos que la utilización de este último tipo de aglutinantes debe atribuirse a reposiciones de las restauraciones.

Por tanto, aunque no de forma concluyente, pero sí con bastante fiabilidad, pensamos que los aglutinantes utilizados en origen debieron ser la goma arábiga y la cola animal, se trata pues de un temple magro. Posteriormente, en sucesivas intervenciones de restauración, se utilizarían aceite de linaza y resinas diterpénicas, que podrían haber impregnado en ocasiones los estratos originales internos.

\section{Conclusiones}

Debemos insistir en que, como la mayoría de los especialistas indican, existe una gran dificultad en la identificación de las zonas originales en el Oratorio de la Madraza, ya que la obra presenta numerosas intervenciones que la enmascaran. Por lo tanto, y puesto que todavía nos falta por completar la información obtenida con nuevos estudios que pretendemos desarrollar durante la fase de la restauración, en este momento, las afirmaciones deben hacerse con cierta cautela en lo que se refiere a la extensión de la policromía original conservada, aunque no así en los resultados obtenidos en la identificación de materiales, ya que éstos son concluyentes y nos confirman la validez de la metodología utilizada.

La mayor parte de la policromía aparente es casi con toda probabilidad consecuencia de la restauración, pero la calidad del relieve, las características de los morteros y la confirmación de la existencia de capas subyacentes de policromía original en algunas de las muestras analizadas, nos indican que pueden conservarse restos considerables de yeserías originales.

En más de treinta estratigrafías se han identificado capas de policromía original, que están ocultas por las diferentes intervenciones (enjalbegados o capas pictóricas posteriores). La realización de catas en la próxima fase del estudio nos permitirá establecer un criterio de valoración que nos oriente sobre la cantidad de policromía original conservada en capas subyacentes.

De forma global debemos resaltar la presencia de barita en un número muy considerable de muestras, lo que puede asociarse al 
uso del pigmento blanco fijo, sulfato de bario de fabricación industrial que comienza a utilizarse en la segunda mitad del siglo XVIII. La presencia de este pigmento así como la de otros que corresponden claramente a esta misma etapa (blanco de zinc, litopón, blanco de titanio, amarillo de bario, etc.) nos permiten descartar estos estratos como originales.

Finalmente podemos avanzar que los pigmentos originales son: para el color blanco, yeso; para el azul, la azurita; para el negro, el negro de carbón, y para el rojo, el bermellón, mezclado en ocasiones con minio o con tierra roja, así como que los aglutinantes originales utilizados son, muy probablemente, la goma arábiga y la cola proteínica, si bien, en este caso, el nivel de deterioro y la posibilidad de contaminación de los estratos subyacentes por la impregnación de los aglutinantes utilizados para los repintes, nos impiden por el momento descartar categóricamente otras opciones.

Recibido: 04/12/2008

Aceptado: 24/06/2009 